\title{
Perfil inmunohistoquímico del mixoma odontogénico, con énfasis en marcadores de agresividad tumoral y microdensidad vascular
}

\author{
Immunohistochemical profile of odontogenic myxoma, with a focus \\ on microvascular density and tumor aggressiveness markers
}

Perfil imuno-histoquímico do mixoma odontogênico, com ênfase em marcadores de agressividade tumoral e microdensidade vascular

\author{
Zaira Gómez-Herrera' ORCID: 0000-0002-1925-0291 \\ Celeste Sánchez-Romero² ORCID: 0000-0001-5365-2692 \\ Gabriela Vigil-Bastitta² ORCID: 0000-0002-0617-1279 \\ Vanesa Pereira-Prado² ORCID: 0000-0001-7747-6718 \\ Estefania Sicco² ORCID: 0000-0003-1137-6866 \\ Omar Tremillo-Maldonado ${ }^{3}$ ORCID:0000-0002-2798-1596 \\ Ronell Bologna-Molina² ORCID:0000-0001-9755-4779
}

\section{Resumen}

Con el fin de tener una mayor comprensión sobre el comportamiento biológico del mixoma odontogénico (MO), se realizó inmunohistoquímica en 31 muestras, utilizando marcadores relacionados con mecanismos de progresión tumoral (adhesión, angiogénesis, apoptosis, inflamación y proliferación celular). El epitelio odontogénico fue detectado en cuatro muestras mediante CK19 y CD138, este último, mostró expresión baja en matriz extracelular (MEC) y alta en las células tumorales. La microdensidad vascular (MDV) media fue de 7.51 y 5.35 vasos marcados con CD34 y VEGF-A respectivamente. Una alta expresión de Orosomucoide-1 y Mast Cell Tryptase se observó células tumorales y en MEC. El MO mostró negatividad para Calretinina. Este perfil inmunohistoquímico, la baja expresión para Ki-67, Bcl-2 y p53, y la relativamente baja $\mathrm{MDV}$, sugieren que la actividad proliferativa, anti-apoptótica o angiogénica no representan los principales mecanismos de crecimiento del $\mathrm{MO}$, los cuales podrían estar asociados a eventos como inmunomodulación y degradación de la MEC.

Palabras clave: mixoma odontogénico, inmunohistoquímica, marcadores tumorales, angiogénesis.

\footnotetext{
Departamento de Atención a la Salud, Universidad Autónoma Metropolitana (UAM), México.

Área de Patología Molecular Estomatológica, Facultad de Odontología, Universidad de la República, Uruguay.

Universidad Autónoma Metropolitana de México, unidad Xochimilco, México.
}

Fecha recibido: 25/07/2019 - Fecha aceptado 9/12/2019 


\section{Abstract}

Immunohistochemistry tests were performed in 31 samples to elucidate the biological behavior of the odontogenic myxoma (OM), using markers related to mechanisms of tumor progression (adhesion, angiogenesis, apoptosis, inflammation and cell proliferation). Odontogenic epithelium was detected in four samples with CK19 and CD138; the latter had a low expression in the extracellular matrix (ECM) and a high expression in tumor cells. The mean microvascular density (MVD), assessed with CD34 and VEGF-A, was 7.51 and 5.35 blood vessels. A high expression of orosomucoid-1 and mast cell tryptase was observed in tumor cells and ECM, while calretinin was negative. The immunohistochemical profile mentioned above, as well as the low expression of $\mathrm{Ki}$ $67, \mathrm{Bcl}-2$ and $\mathrm{p} 53$ and the relatively low MVD, suggest that the proliferative, antiapoptotic and angiogenic activities do not represent the main growing mechanisms of OM, which could be associated to other events, such as immunomodulation and ECM degradation.

Keywords: odontogenic myxoma, immunohistochemistry, tumor markers, angiogenesis.

\section{Introducción}

Los tumores odontogénicos (TO) constituyen un espectro de lesiones que afecta principalmente a los huesos gnáticos, e incluye desde proliferaciones hamartomatosas hasta neoplasias benignas y tumores malignos ${ }^{(1,2)}$. El mixoma odontogénico (MO) es un TO mesenquimal benigno (con o sin epitelio odontogénico), sin embargo, presenta un comportamiento agresi-

\section{Resumo}

Para melhor compreensão do comportamento biológico do mixoma odontogênico (MO), imuno-histoquímica foi realizada em 31 amostras, utilizando marcadores relacionados aos mecanismos de progressão tumoral (adesão, angiogênese, apoptose, inflamação e proliferação celular). Epitélio odontogênico foi detectado em quatro amostras por CK19 e CD138, o último mostrou baixa expressão na matriz extracelular (MEC) e alta em células tumorais. A microdensidade vascular (MDV) média foi de 7.51 e 5.35 vasos marcados com CD34 e VEGF-A, respectivamente. Uma alta expressão de Orosomucoide-1 e Mast Cell Tryptase foi observada nas células tumorais e na MEC. O MO mostrou negatividade para Calretinina. $\mathrm{O}$ perfil imuno-histoquímico mencionado acima, a baixa expressão de Ki-67, Bcl-2 e p53 e a relativamente baixa $\mathrm{MDV}$, sugerem que a atividade proliferativa, anti-apoptótica ou angiogênica não representam os principais mecanismos de crescimento do $\mathrm{MO}$, os quais poderiam estar associados com eventos como imunomodulação e degradação da MEC.

Palavras-chave: mixoma odontogênico, imuno-histoquímica, marcadores tumorais, angiogênese. 
presenta como un aumento de volumen de crecimiento lento, generalmente asintomático ${ }^{(6)}$, por lo que puede alcanzar grandes dimensiones al momento del diagnóstico. Radiográficamente el aspecto puede ser unilocular (lesiones pequeñas), o más comúnmente multilocular (clásicamente descrito como "raqueta de tenis") (3). Microscópicamente, el MO se caracteriza por la presencia de células fusiformes a estrelladas dispersas en una abundante matriz mixoide compuesta principalmente por glicosaminoglicanos, pudiendo presentar o no islas de epitelio odontogénico, la presencia de fibras de colágeno es variable ${ }^{(3,46,)}$.

A la fecha existen pocos trabajos que describan los componentes moleculares del $\mathrm{MO}$, por lo cual, el objetivo de este trabajo es establecer un perfil inmunohistoquímico de diversos marcadores tumorales y analizar la microdensidad vascular (MDV) con el fin de discutir sus posibles implicaciones en el comportamiento biológico del MO.

\section{Metodología}

\section{Selección de la muestra}

Se incluyeron 31 casos de $\mathrm{MO}$ mandibulares obtenidos del Área de Patología Molecular de la Facultad de Odontología de la Universidad de la República (Uruguay). Dichas muestras se obtuvieron mediante biopsia incisional, fueron fijadas en formaldehido al 10\% y posteriormente se incluyeron en bloques de parafina. Este trabajo fue aprobado por el Comité de Ética de la Facultad de Odontología de la Universidad de la República, número de protocolo: 091900000113-14. Se obtuvo el consentimiento informado de todos los participantes incluidos en el estudio, al momento de la toma de biopsia.

\section{Inmunohistoquímica}

Se realizaron cortes de $3 \mu \mathrm{m}$ y se colocaron sobre laminillas, los cortes se desparafinaron en xilol y posteriormente se hidrataron mediante baños en concentraciones decrecientes de alcohol.
Para la recuperación antigénica se utilizó una solución de citrato de ( $\mathrm{pH}$ 6.2) en olla de presión-horno de microondas a máxima potencia por un minuto. Para el bloqueo de la peroxidasa endógena, se utilizó peróxido de hidrógeno al $0,9 \%$, durante 5 minutos. Los anticuerpos primarios para fueron incubados durante una hora (Tabla 1).

\section{Tabla 1. Datos de los anticuerpos utilizados en este estudio}

\begin{tabular}{|c|c|c|}
\hline Anticuerpo & Casa Comercial & Dilución \\
\hline CK19 & GeneTex & $1: 100$ \\
\hline CD138 & Biocare & $1: 100$ \\
\hline Calretinina & Dako & $1: 100$ \\
\hline Orosomucoide-1 & Abcam & $1: 100$ \\
\hline Mast Cell Tryptase & Dako & $1: 100$ \\
\hline BCl-2 & Biocare & $1: 50$ \\
\hline Ki-67 & Bio SB & $1: 50$ \\
\hline p53 & Bio SB & $1: 200$ \\
\hline VEGF-A & GeneTex & $1: 100$ \\
\hline CD34 & Dako & $1: 50$ \\
\hline
\end{tabular}

Posteriormente, los cortes se incubaron con el anticuerpo secundario biotinilado anti-ratón/ anti conejo y con el complejo streptavidina/peroxidasa (LSA-B+Dako Corporation, Carpintería CA, USA) por 30 minutos cada uno. La reacción se visualizó con sustrato de 3,3'-diaminobenzidinaH2O2 (Dako Corporation, Carpintería, CA, USA). Por último, los cortes fueron contrastados con hematoxilina de Mayer.

Como controles positivos se utilizaron: mucosa oral para CK19 y CD138; cáncer de mama para Calretinina; carcinoma oral para Orosomucoide-1 y p53; intestino para Mast Cell Tryptase, CD34 y VEGF-A; y amígdala Bcl-2 y Ki-67.

\section{Análisis microscópico}

Las reacciones fueron consideradas positivas al observar marcación marrón celular (células tumorales, endoteliales o de islas de epitelio odontogénico), o en la matriz extracelular (MEC). Los patrones de marcación (nuclear, 
citoplasmática, membranosa o de $\mathrm{MEC)}$ ) varían con cada tipo de anticuerpo.

Proteinas evaluadas en islas de epitelio odontogénico: CK19 y CD138

Cada corte fue visualizado por completo bajo un objetivo de 10x para identificar las áreas de positividad y, posteriormente, a 40x para confirmar que se tratara de epitelio odontogénico. El resultado se expresó como positivo o negativo.

Proteinas evaluadas en células tumorales $y$ matriz extracelular: CD138, Calretinina, Orosomucoide-1, Mast Cell Tryptase, Bcl-2, Ki67, p53 y VEGF-A.

En un microscopio óptico, se visualizaron los cortes completos bajo el objetivo de 40x para determinar un porcentaje de inmunoexpresión en la totalidad del tejido tumoral presente en la laminilla. Los porcentajes se clasificaron en 4 grupos: $0 \%$, expresión negativa; de $1 \%$ a $10 \%$, expresión baja; $11 \%$ a $50 \%$, expresión moderada y mayor de $50 \%$ expresión alta ${ }^{(7)}$.

Proteinas evaluadas en vasos sanguineos: VEGF-A y CD34.

Para determinar la MDV, se empleó el método descrito por Weidner et al. Los tejidos fueron visualizados inicialmente bajo un objetivo de $10 \mathrm{x}$, para identificar 3 zonas con la mayor concentración de vasos positivos (hot spots), en las cuales, bajo el objetivo de 40x se realizó el contaje de vasos manualmente. Finalmente se calculó la media del número de vasos en los cinco campos de cada muestra ${ }^{(8)}$.

\section{Resultados}

Epitelio odontogénico positivo para CK19 y CD138 fue detectado en cuatro casos. Adicionalmente CD138 mostró una inmunoexpresión alta en las células tumorales de la mayoría de las muestras (74.2\%) y una expresión baja en la MEC en el 38.7\% de los casos (Figura $1 \mathrm{~A}, \mathrm{~B}$ ). Calretinina fue negativa en todas las muestras de MO. Por el contrario, fue observada alta inmunopositividad de Orosomucoide-1 en células tumorales y MEC en el 100\% de los casos, mientras que Mast Cell Tryptase pudo observarse en forma de gránulos mastocitos, focalmente en la MEC y con una alta expresión en las células tumorales $96.7 \%$ de los casos $(n=30)$ (Figura 2 A, D) (Figura 2 C, E, F).

Una baja marcación de Bcl-2 (citoplasmática), Ki-67 (nuclear) y p53 (nuclear) fue observada en las células tumorales $(n=18,58.1 \% ; n=28$, $90.3 \%$ y $n=12,38.7 \%$ respectivamente).

CD34 y VEGF-A fueron positivas en las células endoteliales (vasos sanguíneos). Adicionalmente, VEGF-A mostró una expresión predominantemente alta en las células tumorales del MO ( $\mathrm{n}=24,77.5 \%$ ) (Tabla 2). La MDV fue de 7.51 vasos con CD34 y 5.35 vasos con VEGFA (Figuras 1C y 2D).

La mayoría de las proteínas que fueron detectadas en células tumorales y en MEC tuvieron una inmunoexpresión alta, a excepción de Bcl2. La tabla 3 muestra el patrón, nivel y distribución de inmunoexpresión de cada proteína en los casos de MO.

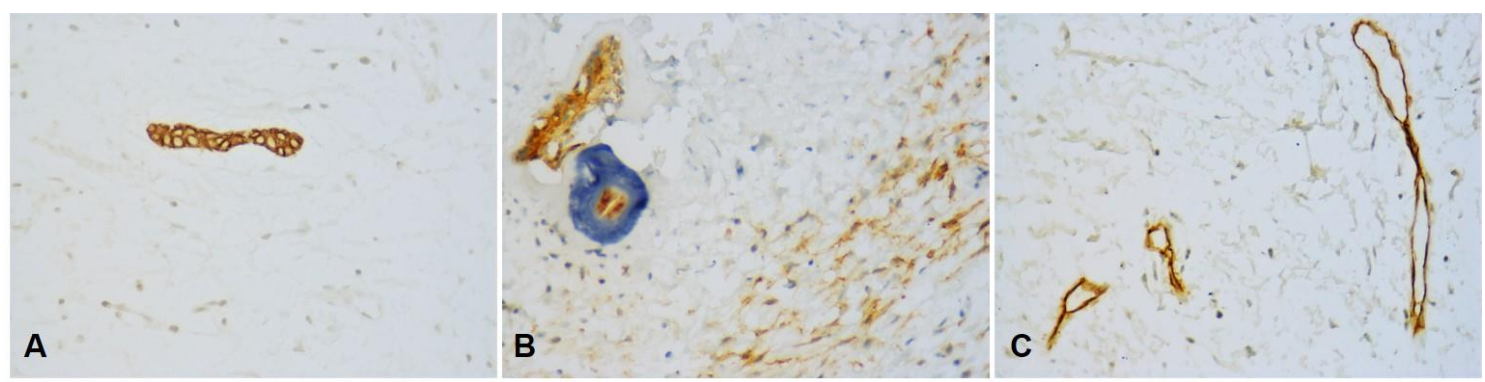

Fig. 1. Marcadores inmunohistoquímicos en mixoma odontogénico. Pequeñas islas de epitelio odontogénico mostraron positividad para CK19 (A), y CD138 (B), la cual también se expresó en células tumorales y MEC. Únicamente las células endoteliales de los vasos sanguíneos mostraron positividad para CD34 (C). (Magnificación original, A-C: 400x). 


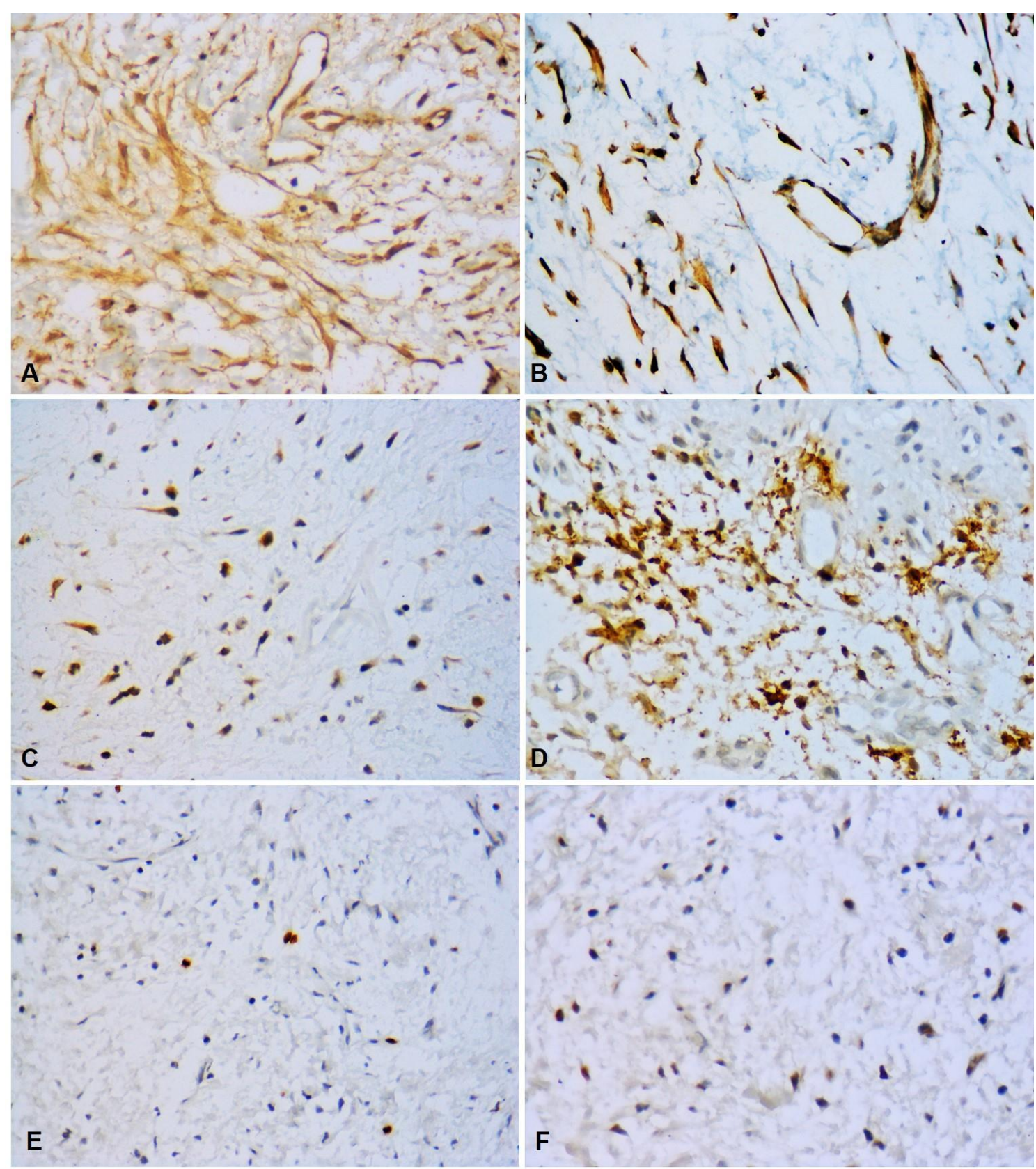

Fig. 2. Perfil inmunohistoquímico del mixoma odontogénico. Alta inmunopositividad de Orosomucoide-1 en células tumorales, células endoteliales y MEC (A). Expresión de VEGF-A en células tumorales y células endoteliales (B). Baja marcación de Bcl-2 en algunas células tumorales (C). Mast Cell Tryptase intensamente positiva con aspecto granular en las células tumorales, mastocitos y MEC (D). Escasos núcleos positivos para Ki-67 (E) y p 53 (F). (Magnificación original, A, B, C, E, F: 400x; D: 600x). 
Tabla 2. Distribución de la expresión de los marcadores dentro de los componentes del mixoma odontogénico.

\begin{tabular}{|l|c|c|c|c|}
\hline Proteína & CT & MEC & E0 & VS \\
\hline CK19 & - & - & + & - \\
\hline CD138 & + & + & + & - \\
\hline Calretinina & - & - & - & - \\
\hline Orosomucoide-1 & + & + & - & + \\
\hline Mast Cell Tryptase & + & - & - & - \\
\hline BCl-2 & + & - & - & - \\
\hline Ki-67 & + & - & - & - \\
\hline p53 & + & - & - & - \\
\hline VEGF-A & + & - & - & + \\
\hline CD34 & - & - & - & + \\
\hline
\end{tabular}

Abreviaciones. CT: células tumorales, MEC: matriz extracelular, EO: epitelio odontogénico, VS: vasos sanguíneos.

Tabla 3. Número y porcentaje de casos de acuerdo al nivel de inmunoexpresión que presentaron.

$\begin{array}{lcccc}\text { Proteína } & \text { Negativa } & \text { Baja } & \text { Moderada } & \text { Alta } \\ \text { CD138 en CT } & 0 & 5 & 3 & 23 \\ & (0 \%) & (16.1 \%) & (9.7 \%) & (74.2 \%) \\ \text { CD138 en MEC } & 4 & 12 & 7 & 8 \\ & (12.9 \%) & (38.7 \%) & (22.6 \%) & (25.8 \%) \\ \text { Orosomucoi- } & 0 & 0 & 0 & 31 \\ \text { de-1 en CT y } & (0.0 \%) & (0.0 \%) & (0.0 \%) & (100 \%) \\ \text { MEC } & & & & \\ \text { Mast Cell } & 0 & 0 & 1 & 30 \\ \text { Tryptase en CT, } & (0.0 \%) & (0.0 \%) & (3.3 \%) & (96.7 \%) \\ \text { My MEC } & & & & \\ \text { VEGF-A* en CT } & 0 & 0 & 7 & 23 \\ & (0.0 \%) & (0.0 \%) & (23.3 \%) & (76.7 \%) \\ \text { Bcl-2 } & 2 & 18 & 9 & 2 \\ & (6.5 \%) & (58 \%) & (29 \%) & (6.5 \%) \\ \text { Ki-67 } & 0 & 30 & 1 & 0 \\ & (0.0 \%) & (96.7 \%) & (3.3 \%) & (0.0 \%) \\ \text { p53 } & 12 & 12 & 6 & 1 \\ & (38.7 \%) & (38.7 \%) & (19.4 \%) & (3.2 \%) \\ \text { VEGF-A } & 0 & 0 & 7 & 24 \\ & (0.0 \%) & (0.0 \%) & (22.5 \%) & (77.5 \%)\end{array}$

Abreviaciones. CT: células tumorales, M: mastocitos. MEC: matriz extracelular.

*La expresión de VEGF-A en células endoteliales fue cuantificada como microdensidad vascular (ver en sección de resultados).

\section{Discusión}

Se ha propuesto un origen odontogénico del MO con base en la presencia ocasional de pequeñas islas de epitelio odontogénico, su localización en mandíbula y maxilares, y su similitud histomorfológica con la porción mesenquimal del germen dentario ${ }^{(9)}$.

En el presente estudio, en el $13.3 \%$ de la muestra se encontraron islas de epitelio odontogénico positivas para CD138 y CK19, coincidiendo con estudios anteriores en los que usando CK19, se detectaron islas epiteliales en el $4.8 \%$ y $14 \%$ de los casos ${ }^{(9,10)}$. Adicionalmente, nuestros resultados corroboran la utilidad de CD138 como un marcador alternativo de epitelio odontogénico en el MO.

Una alta expresión de CD138 fue observada en células tumorales en la mayoría de los casos $(73.30 \%)$, mientras que, en la MEC, la inmunoexpresión fue principalmente baja (46.70\% de los casos). Contrario a nuestros resultados, en el estudio de Etemad-Moghadam et al. (2017) CD138 fue negativo en todas sus muestras ${ }^{(11)}$. El papel fisiológico de CD138, en conjunto con la MEC es la participación en la inducción y regulación de la proliferación mediante la interacción con familias de factores de crecimiento ligados a la heparina ${ }^{(12)}$; además, interactúa con otros componentes de la MEC ${ }^{(13)}$, algunos de los cuales (colágeno tipo I, fibronectina y tenascina) forman parte de la MEC del $\mathrm{MO}{ }^{(14)}$, sugiriendo así que CD138, además de la función de mantenimiento estructural propia de una proteína de adhesión epitelial, al ser liberada en la MEC, podría estar involucrada en diversas vías de señalización al interactuar con factores de crecimiento y otras moléculas presentes en la MEC.

A pesar de que la expresión de calretinina ha sido descrita en ameloblastomas, y ha sido asociada a la producción de esmalte en germen dentario, en nuestro estudio resultó negativa en $\mathrm{MO}$, al igual que en trabajos previos ${ }^{(15,16)}$. Esta ausencia de calretinina puede ser explicada por 
el origen mesenquimal del $\mathrm{MO}$, a diferencia del ameloblastoma y el órgano del esmalte ${ }^{(17,18)}$. Un estudio previo determinó la MDV en el MO mediante CD34, mostrando resultados similares a los nuestros ${ }^{(7)}$. El ameloblastoma presenta una mayor MDV comparado con otros tumores y quistes odontogénicos, lo que sugiere una asociación con un comportamiento más agresivo ${ }^{(19,20)}$. En nuestro estudio, el MO mostró una MDV aproximadamente 5 veces menor que los ameloblastomas del estudio de Seifi et al. (2001), sugiriendo que, en el MO, la angiogénesis podría contribuir limitadamente al potencial de crecimiento tumoral ${ }^{(19)}$.

En MO, VEGF-A (una de las principales proteínas señalizadoras para la angiogénesis), se expresó en células endoteliales y en las células tumorales. Investigaciones recientes sugieren que la inmunoexpresión de VEGF-A en el epitelio de quistes odontogénicos y TO epiteliales como el ameloblastoma, influye en la proliferación epitelial mediante un mecanismo autócrino; mientras que la actividad anigiogénica mediada por un mecanismo parácrino ${ }^{(7,21)}$. Sin embargo, en el único estudio describiendo la expresión de VEGF-A en MO, se ha asociado principalmente a mecanismos angiogénicos de este tumor ${ }^{(7)}$. En las muestras de MO analizadas, se observó una inmunoexpresión alta de Orosomucoide-1 en células tumorales y MEC. Confirmando los hallazgos de los dos estudios que reportaron la sobreexpresión de Orosomucoide-1 en $\mathrm{MO}$ mediante inmunohistoquímica y preoteómica (7,22).

Macroscópicamente, el MO tiene la apariencia de una masa mucoide altamente viscosa, la cual, de acuerdo a nuestros resultados, podría deberse en parte a la presencia de Orosomucoide-1 (que es una mucoproteína) en la MEC. Es ampliamente aceptado que la viscosidad estructural del MO, le confiere la habilidad de infiltrar e invadir ${ }^{(7)}$. Por otra parte, García Muñoz et al. (2012), propusieron que la sobreexpresión de Orosomucoide-1 en el MO podría jugar un papel importante en el crecimiento y potencial de invasión de las células tumorales, al inhibir respuesta inmune antitumoral ${ }^{(22)}$.

Se ha demostrado que Orosomucoide-1 participa en la regulación e inducción de VEGF-A. ${ }^{(23,24)}$ De esta manera, la sobreexpresión de ambas proteínas en las células tumorales y endoteliales del MO podría indicar una interacción entre Orosomucoide-1 y VEGF-A en este tumor, indicando con un rol colaborativo proangiogénico ${ }^{(7)}$.

En el presente estudio, la mayoría de los casos expresaron inmunopositividad alta para Mast Cell Tryptase (96,70\%) en células tumorales, mastocitos y MEC. Un estudio previo en siete casos de $\mathrm{MO}$, reportó la ausencia de expresión de Mast Cell Tryptase; sin embargo, el tamaño de la muestra podría no ser representativa (25). Otra pesquisa en una muestra mayor (62 casos), encontró la presencia de Mast Cell Tryptase en el 72.6\% de los MO localizándose principalmente en los mastocitos, los cuales contienen grandes cantidades de triptasa activa almacenada que al ser liberada es capaz de degradar fibronectina (uno de los componentes de la MEC del MO), lo que sugiere que, en parte, la degradación de la MEC podría estar mediada por la liberación de Mast Cell Tryptase. ${ }^{(9,26)}$ Adicionalmente, existe una asociación relación entre la presencia de mastocitos y Mast Cell Tryptase con la reabsorción ósea en quistes odontogénicos ${ }^{(27)}$, y a pesar de que la incidencia de mastocitos en el $\mathrm{MO}$ es variable entre algunos estudios, se ha descrito que estas células frecuentemente se distribuyen adyacente a trabéculas óseas residuales ${ }^{(28)}$. Dichos hallazgos sugieren que la degradación de la MEC y procesos de remodelación ósea mediante la triptasa, contribuye de manera importante en el potencial de invasión del $\mathrm{MO}^{(27,28)}$.

El desbalance entre los mecanismos de apoptosis y proliferación celular representan importantes alteraciones moleculares tumorigénicas en diversas neoplasias.

En el presente estudio fueron evaluadas p53, Bcl-2 y Ki-67, proteínas relacionadas con pro- 
cesos apoptóticos, anti apoptóticos y de proliferación celular respectivamente. Se ha demostrado que la apoptosis inducida por $\mathrm{p} 53$ puede ser bloqueada por $\mathrm{Bcl}-2{ }^{(29)}$. Al igual que p53, Bcl-2 mostró una baja inmunoexpresión en la mayoría de los $\mathrm{MO}$, lo que coincide con reportes previos ${ }^{(9,30)}$. De igual manera, Ki-67 mostró un bajo índice proliferativo en nuestros casos coincidiendo con diversos autores ${ }^{(9,30)}$, Estos resultados podrían indicar que, debido al bajo nivel de actividad anti-apoptótica y proliferativa en este tumor, estos no serían los principales mecanismos asociados a el potencial de crecimiento y agresividad del MO.

\section{Conclusiones}

La marcación CK19 y CD138 es útil para detectar epitelio odontogénico en el MO. La alta expresión de Orosomucoide-1 en la MEC lo indica como un componente estructural que contribuye a su viscosidad y, por ende, facilitando la invasión. Orosomucoide-1 también mostró un patrón semejante a VEGF-A en las células tumorales y endoteliales, sugiriendo una actividad colaborativa pro-angiogénica, posiblemente implicada en el desarrollo tumoral. La actividad anti-apoptótica y proliferativa no se perfilan como mecanismos cruciales en el comportamiento agresivo del MO. Sin embargo, la degradación de la MEC y la reabsorción ósea mediada por Mast Cell Tryptase, podrían ser importantes para el alto potencial invasivo del MO.

\section{Contribución de autoría}

1. Concepción y diseño del estudio

2. Adquisición de datos

3. Análisis de datos

4. Discusión de los resultados

5. Redacción del manuscrito

6. Aprobación de la versión final del manuscrito

ZGH, CSR y RBM han contribuido en $1,2,3,4,5$ y 6 .

GVB y VPP han contribuido en 3, 4, 5 y 6.

ES ha contribuido en 4,5 y 6.

OTM ha contribuido en 1, 2, y 3 .

\section{Referencias}

1. El-Naggar Chan JKC, Grandis JR, Takata T, Slootweg P, editors. WHO classification of Head and Neck Tumours. Chapter 8: Odontogenic and maxilofacial bone tumours. 4th edition, IARC: Lyon 2017, p.205-260.

2. Mosqueda-Taylor A, Ledesma-Montes C, Caballero-Sandoval S, Portilla-Robertson J, RuízGodoy RLM, Meneses-García A. Odontogenic tumors in México. A collaborative retrospective study of 349 cases. Oral Surg Oral Med Oral Pathol Oral Radiol Endod. 1997; 84 (6): 6725.

3. Wright JM, Soluk-Tekkesin M. Odontogenic tumors: where are we in 2017? J Istanb Univ Fac Dent. 2017; 51 (3 Suppl 1): s10-30.

4. Gonzalez-Galvan MC, Mosqueda-Taylor A, Bologna-Molina R, Setien-Olarra A, Marichalar-Mendia X, Aguirre-Urizar JM. Evaluation of the osteoclastogenic process associated with RANK / RANK-L / OPG in odontogenic myxomas. Med Oral Patol Oral Cir Bucal. 2018; 23 (3): e315-9.

5. Kanitkar S, Kamat M, Tamagond S, Vareakr A, Datar U. Peripheral odontogenic myxoma in a 12-year-old girl: a rare entity. J Korean Assoc Oral Maxillofac Sur. 2017;43: 178.81. doi: 10.5125/jkaoms.2017.43.3.178 
6. Chrcanovic BR, Gomez RS. Odontogenic myxoma: an updated analysis of 1,692 cases reported in the literature. Oral Dis. 2019; 25: 676-83. doi: 10.1111/odi.12875

7. Bologna-Molina R, Mosqueda-Taylor A, Dominguez-Malagon $\mathrm{H}$, Salazar-Rodriguez $\mathrm{S}$, Tapia G, Gonzalez-Gonzalez R, Molina-Frechero $\mathrm{N}$. Immunolocalization of VEGF-A and orosomucoid-1 in odontogenic myxoma. Rom J Morphol Embryol. 2015; 56 (2): 465-73.

8. Weidner N, Semple JP, Welch WR, Folkman $\mathrm{J}$. Tumor angiogenesis and metastasis - correlation in invasive breast carcinoma. $\mathrm{N}$ Engl J Med. 1991; 32: 1-8.

9. Martínez-Mata G, Mosqueda-Taylor A, Carlos-Bregni R, Paes de Almeida O, ContrerasVidaurre E, Vargas PA, Cano-Valdéz AM, Domínguez-Malagón H. Odontogenic myxoma: clinico-pathological, immunohistochemical and ultrastructural findings of a multicentric series. Oral Oncol. 2007; 44: 601-7. doi:10.1016/j.oraloncology 2007.08.009.

10. Lombardi T, Lock C, Samson J, Odell EW. S100, alpha-smooth muscle actin and cytokeratin 19 immunohistochemistry in odontogenic and soft tissue myxomas. J Clin Pathol. 1995; 48: 759-62.

11. Etemad-Moghadam S, Alaeddinni M. A comparative study of syndecan-1 expression in different odontogenic tumors. J Oral Biol Craniofac Res. 2017; 7: 23-6. doi: 10.1016/j. jobcr.2016.11.001

12. Bologna-Molina R, Salazar-Rodríguez $S$, Bedoya-Borella AM, Carreon-Burciaga RG, Tapia-Repetto G, Molina-Frechero N. A histopathological and immunohistochemical analysis of ameloblastic fibrodentinoma. Case Rep Pathol. 2013; 2013:604560. doi: $10.1155 / 2013 / 604560$

13. Bologna-Molina R, Mosqueda-Taylor A, López-Corella E, Paes de Almeida O, CarrascoDaza D, Farfán-Morales JE, Molina-Frechero N, Damián-Matsumura P. Comparative expression of syndecan-1 and Ki-67 in peripheral and desmoplastic ameloblastomas and ameloblastic carcinoma. Pathol Int. 2009; 59: 229-33.

14. Manne RK, Kumar VS, Venkata Sarath P, Anumula L, Mundlapudi S, Tanikonda R. Odontogenic myxoma of the mandible.
Case Rep Dent. 2012; 2012: 214704. doi: $10.1155 / 2012 / 214704$

15. Alaeddini M, Etemad-Moghadam S, Baghaii F. Comparative expression of calretinin in selected odontogenic tumours: a possible relationship to histogenesis. Histopathology. 2008; 52 (3): 299-304.

16. Terracciano LM, Mhawech P, Suess K, D'Armiento M, Lehmann FS, Jundt G, Moch H, Sauter G, Mihatsch MJ. Calretinin as a marker for cardic myxoma. Diagnostic and histogenetic considerations. Am J Clin Pathol. 2000; 114 (5): 754-9.

17. Mistry D, Altini M, Coleman HG, Ali H, Maiorano E. The spatial and temporal expression of calretinin in developing rat molars (Rattus norvegicus). Arch Oral Biol. 2001; 46 (10): 973-81.

18. Altini M, Coleman H, Doglioni C, Favia G, Maiorano E. Calretinin expression in ameloblastomas. Histopatholohy. 2000; 37 (1): $27-$ 32.

19. Seifi S, Shafaie S, Ghadiri S. Microvessel density in follicular cysts, keratocystic odontogenic tumours and ameloblastomas. Asian Pacific J Cancer Prev. 2001; 12 (2): 351-56.

20. Treville Pereira, Shashibhushan Dodal, Avinash Tamgadge, Sudhir Bhalerao, Sandhya Tamgadge. Quantitative evaluation of microvessel density using CD34 in clinical variants of ameloblastoma: An immunohistochemical study. J Oral Maxillofac Pathol. 2016; 20 (1): 51-8. Doi: 10.4103/0973-029X.180929

21. Gupta B, Chandra S, Singh A, Sah K, Raj V, Gupta V. The role of vascular endothelial growth factor in proliferation of odontogenic cysts and tumors: An immunohistochemical study. Dent Res J. 2016; 13 (3): 256-63.

22. García-Muñoz A, Rodríguez MA, BolognaMolina R, Cázares-Raga FE, Hernández-Hernández FC, Farfán-Morales JE, Trujillo JJ, Licéaga-Escalera C, Mendoza-Hernández G. The orosomucoid 1 protein (a1 acid glycoprotein) is overexpressed in odontogenic myxoma. Proteome Sci. 2012; 10 (1): 49.

23. Irmak $S$, Oliveira-Ferrer L, Singer BB, Ergun $S$, Tilki D. Pro-angiogenic properties of orosomucoid (ORM). Exp Cell Res. 2009; 315 (18): 3201-09. 
24. Ligresti G, Aplin AC, Dunn BE, Morishita A, Nicosia RF. The acute phase reactant orosomucoid-1 is a bimodal regulator of angiogenesis with time- and context dependent inhibitory and stimulatory properties. Plos One. 2012; 7 (8): e41387. doi: 10.1371/journal. pone. 0041387 .

25. Kouhsoltani M, Halimi M, Dibazar S. A positive correlation between immunohistochemical expression of CD31 and mast cell tryptase in odontogenic tumor. Pol J Pathol. 2015; 66 (2): $170-5$.

26. Fajardo I, Pejler G. Human mast cell ß-tryptase is a gelatinase. J Immunol. 2003; 171 (3): 1493-9.
27. Huang S, Lu F, Chen Y, Huang B, Liu M. Mast cell degranulation in human periodontitis. J Periodontol. 2013; 84 (2): 248-55.

28. De Assis Caldas Pereira F, Araújo Silva Gurgel CA, Ramos EA, Vidal MT, Pinheiro AL, Jurisic V, Sales CB, Cury PR, dos Santos JN. Distribution of mast cell in benign odontogenic tumors. Tumor Biol. 2012; 33 (2): 455-61.

29. Iezzi G, Piatelli A, Rubini C, Artese L, Fiorini $\mathrm{M}$, Carinci F. MIB-1, Bcl-2 and p53 in odontogenic myxomas of the jaws. Acta Otorhinolaryngol Ital. 2007; 27 (5): 237-42.

30. Bast B, Pogrel MA, Regezi JA. The expression of apoptotic proteins and matrix metalloproteinases in odontogenic myxomas. J Oral Maxillofac Surg. 2003; 61 (12): 1463-6.

Celeste Sanchez Romero: csr_90@hotmail.com 